\title{
Focal Segmental Glomerulosclerosis
}

National Cancer Institute

\section{Source}

National Cancer Institute. Focal Segmental Glomerulosclerosis. NCI Thesaurus. Code C37308.

A renal disorder characterized by sclerotic lesions in the glomeruli. Causes include drugs, viruses, and malignancies (lymphomas), or it may be idiopathic. It presents with asymptomatic proteinuria or nephritic syndrome and it may lead to renal failure. 